Kragujevac Journal of Mathematics

Volume 40(1) (2016), Pages 105-112.

\title{
NOTE ABOUT ASYMPTOTIC BEHAVIOUR OF POSITIVE SOLUTIONS OF SUPERLINEAR DIFFERENTIAL EQUATION OF EMDEN-FOWLER TYPE AT ZERO
}

\author{
MARIJA MIKIĆ ${ }^{1}$
}

\begin{abstract}
We study the asymptotic behavior of solutions of the differential equation of Emden-Fowler type

$$
y^{\prime \prime}-x^{a} y^{\sigma}=0,
$$

where $a \in \mathbb{R}, \sigma>1$. We prove some of theorems on asymptotic properties of solutions and obtain asymptotic formulas for solutions near zero.
\end{abstract}

\section{INTRODUCTION}

In this paper we study very important non-linear second-order differential equation

$$
y^{\prime \prime}-x^{a} y^{\sigma}=0
$$

This equation came first into prominence in connection with the astrophysical researcher Emden. A number of results obtained by Emden in the usual half-intuitive, wholly ingenious fashion of the physicist were made by Fowler, who was then stimulated to continue and give a complete discussion of solutions of this equation for all values of the parameters. The equation has several very interesting physical applications, occurring in astrophysics in the form of the Emden equation and in atomic physics in the form of Fermi-Thomas equation.

Mathematically, the equation has great potential. It is a nontrivial, nonlinear, differential equation with a large class of solutions whose behaviour can be ascertained with astonishing accuracy, despite the fact that the solutions, in general, can't be obtained explicitly.

Key words and phrases. Superlinear Emden-Fowler differential equation, asymptotic behavior of solutions, asymptotic equivalence.

2010 Mathematics Subject Classification. Primary: 34E10. Secondary: 34C41.

Received: December 25, 2015.

Accepted: January 27, 2016. 
The Emden-Fowler type of equation has significant applications in many fields of scientific and technical world and this equation has been investigated by many researchers. This equation was considered, e.g. in $[1-3,5]$.

In $[2,3,5]$ were obtained asymptotic properties of solutions of the Emden-Fowler equation at infinity. In paper [1] was obtained some asymptotic properties of solutions of the Emden-Fowler equation near zero. These papers motivate us to fully examine the behaviour of solutions Emden-Fowler equation near zero in the case when $\sigma>1$ and $a \in \mathbb{R}$.

The Emden-Fowler differential equation $y^{\prime \prime}-x^{a} y^{\sigma}=0$ is said to be superlinear or sublinear according as $\sigma>1$ or $0<\sigma<1$.

Our paper is divided into two parts. In Section 2 we present definition and some lemmas that are very useful for proof of the main theorem in the Section 3. In Section 3 , we proved main theorem and we obtain asymptotic formulas of positive solutions of superlinear Emden-Fowler equation near zero.

\section{Definitions and Auxiliary Results}

Definition 2.1. Two non-trivial functions $y_{1}(x)$ and $y_{2}(x)$ are asymptotically equivalent as $x \rightarrow \infty$ if $y_{1}(x)=y_{2}(x)(1+o(1))$ as $x \rightarrow \infty$.

The asymptotical behavior of solutions of quasi-linear second-order differential equations has been investigated a lot $[2,4]$. Let's consider some results for equation

$$
u^{\prime \prime}-B u^{\prime}+A u-|u|^{k-1} u=0,
$$

where $u=u(t), A=$ const, $B=$ const $>0$, and $k>1$.

In the sequel we will use the following results from [4].

Lemma 2.1. Suppose $A<0$. Then for any non-trivial solution $u(t)$ of $(2.1)$ defined in a neighbourhood (of $+\infty$ ) it holds

$$
u(t)=(C+o(1)) e^{-m t},
$$

as $t \rightarrow+\infty$, with some non-zero constant $C$ and $m=\sqrt{\frac{B^{2}}{4}-A}-\frac{B}{2}>0$. Such a solution exists for any $C \neq 0$.

Lemma 2.2. Suppose $A=0$. Then for any non-trivial solution $u(t)$ of $(2.1)$ defined in a neighbourhood (of $+\infty$ ) it holds

$$
u(t)= \pm\left(\frac{k-1}{B} t\right)^{-\frac{\beta}{2}} \cdot(1+o(1)),
$$

as $t \rightarrow+\infty$, where $\beta=\frac{2}{k-1}>0$. Such a solution exists.

Lemma 2.3. Suppose $A>0$. Then for any non-trivial solution $u(t)$ of $(2.1)$ defined in a neighbourhood $($ of $+\infty$ ) it holds

$$
u(t)= \pm A^{\frac{\beta}{2}}+o(1)
$$


as $t \rightarrow+\infty$, where $\beta=\frac{2}{k-1}>0$. Such a solution exists.

Let's consider some results for equation

$$
u^{\prime \prime}+B_{1} u^{\prime}+A u-|u|^{k-1} u=0,
$$

where $u=u(t), A=$ const, $B_{1}=$ const $>0$, and $k>1$.

Lemma 2.4. Suppose $A<0$. Then for any non-trivial solution $u(t)$ of $(2.2)$ defined in a neighbourhood (of $+\infty)$ it holds

$$
u(t)=(C+o(1)) e^{-M t},
$$

as $t \rightarrow+\infty$, with some non-zero constant $C$ and $M=\sqrt{\frac{B_{1}^{2}}{4}-A}+\frac{B_{1}}{2}>0$. Such a solution exists for any $C \neq 0$.

Lemma 2.5. Suppose $A=0$. Then for any non-trivial solution $u(t)$ of $(2.2)$ defined in a neighbourhood (of $+\infty$ ) it holds

$$
u(t)=(C+o(1)) e^{-B_{1} t},
$$

as $t \rightarrow+\infty$, with some non-zero constant $C$. Such a solution exists for any $C \neq 0$.

Lemma 2.6. Suppose $0<A<\frac{B_{1}^{2}}{4}$. Then for any non-trivial solution $u(t)$ of $(2.2)$ tending to 0 as $t \rightarrow+\infty$ it holds

$$
u(t)=C e^{-m t}(1+o(1))
$$

or

$$
u(t)=C e^{-M t}(1+o(1)),
$$

with some non-zero constant $C, m=\frac{B_{1}}{2}-\sqrt{\frac{B_{1}^{2}}{4}-A}$, and $M=\sqrt{\frac{B_{1}^{2}}{4}-A}+\frac{B_{1}}{2}$. Such solutions exists for any $C \neq 0$.

The reader can find proofs of Lemma 2.1, Lemma 2.2, Lemma 2.3, Lemma 2.4, Lemma 2.5 and Lemma 2.6 in [4].

\section{Asymptotic Behaviour of Positive Solutions of the Superlinear EMDEN-FOWLER EQUATION}

The asymptotical behaviour of solutions of the Emden-Fowler equations was investigated a lot (see references [2,5]). Let's consider some results for superlinear equation

$$
y^{\prime \prime}-x^{a} y^{\sigma}=0
$$

where $y=y(x), x>0$, and $a \in \mathbb{R}$.

We will analyse the asymptotic of positive solutions of (3.1) as $x \rightarrow+0$. In order to prove the next theorem easier to follow, and for completeness of considered cases, we show Figure 1.

Let's comment now Figure 1. The Figure 1 shows that all considered cases for which the asymptotic results of behaviour of positive solutions of differential equations (3.1) 


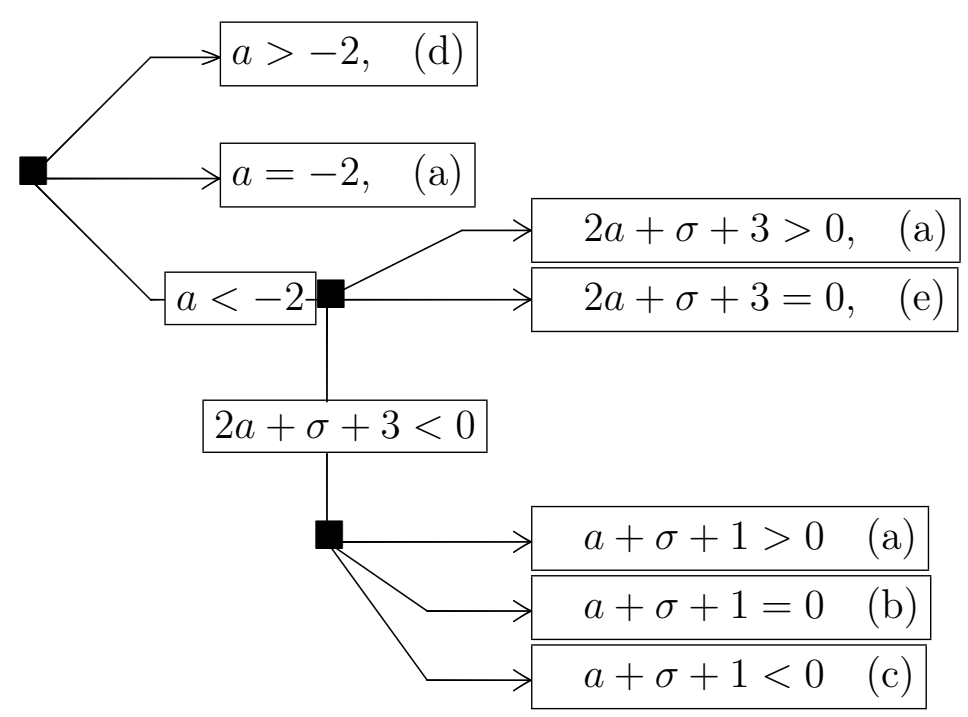

FIGURE 1.

when $x$ tends to zero. It may be noted that the results of behaviour of positive solutions of differential equations (3.1) for each $a \in \mathbb{R}$ and $\sigma>1$, i.e., it is obtained complete asymptotic behaviour of positive solutions of superlinar differential equations of Emden-Fowler type.

All cases shown in Figure 1 are processed in the following theorem.

Theorem 3.1. Suppose $\sigma>1$. Then for any positive solutions $y(x)$ of (3.1) the following statements are true:

a) If $2 a+\sigma+3<0, a<-2, a+\sigma+1>0$ or $a<-2,2 a+\sigma+3>0$ or $a=-2$, then

$$
y(x)=(C+o(1)) \cdot x,
$$

as $x \rightarrow+0$, with some constant $C>0$. Such a solution exists for any $C>0$.

b) If $a<-2$ and $a+\sigma+1=0$, then

$$
y(x)=((a+2) \ln x)^{\frac{1}{a+2}} \cdot x \cdot(1+o(1)),
$$

as $x \rightarrow+0$. Such a solution exists.

c) If $a<-2$ and $a+\sigma+1<0$, then

$$
y(x)=\left(\left(\frac{(a+2)(a+\sigma+1)}{(1-\sigma)^{2}}\right)^{\frac{1}{1-\sigma}}+o(1)\right) \cdot x^{\frac{a+2}{1-\sigma}},
$$

as $x \rightarrow+0$. Such a solution exists.

d) If $a>-2$, then

$$
y(x)=C(1+o(1)),
$$

or

$$
y(x)=C(1+o(1)) \cdot x,
$$


as $x \rightarrow+0$, with some constant $C>0$. Such a solution exists for any $C>0$.

e) If $2 a+\sigma+3=0$, then solutions have parametric form

$$
\begin{aligned}
& x(\tau)=m C_{2}^{2} e^{2 \int\left(\frac{8}{\sigma+1} \tau^{\sigma+1}+\tau^{2}+C_{1}\right)^{-\frac{1}{2}} d \tau} \\
& y(\tau)=n C_{2} \tau e^{\int\left(\frac{8}{\sigma+1} \tau^{\sigma+1}+\tau^{2}+C_{1}\right)^{-\frac{1}{2}} d \tau}
\end{aligned}
$$

where $\left(\frac{m}{n^{2}}\right)^{\frac{\sigma-1}{2}}=1$ and $C_{1}, C_{2}$ are constants, $C_{2}>0$, and $C_{1}$ such that the expression is well-defined.

Proof. The change of variables $x=e^{-t}, y=u e^{-\lambda t}$, where $\lambda=\frac{a+2}{1-\sigma}$ and $u=u(t)$, reduces (3.1) to the form

$$
u^{\prime \prime}-(2 \lambda-1) u^{\prime}+\lambda(\lambda-1) u-u^{\sigma}=0 .
$$

Thus, we reduced (3.1) to (2.1) with $A=\frac{(a+2)(a+\sigma+1)}{(1-\sigma)^{2}}$ and $B=\frac{2 a+\sigma+3}{1-\sigma}$. Note that we have

$$
\frac{B^{2}}{4}-A=\frac{1}{4}
$$

a) To prove this proposition we will use Lemma 2.1, Lemma 2.4 and Lemma 2.5. If $2 a+\sigma+3<0, a<-2, a+\sigma+1>0$, then $A<0$ and $B>0$. From the Lemma 2.1 we have that

$$
u(t)=(C+o(1)) e^{-m t},
$$

as $t \rightarrow+\infty$, with some constant $C>0$ and form (3.2) $m=\frac{1-B}{2}>0$. In this case $m=\frac{a+\sigma+1}{\sigma-1}$. Thus, we obtain

$$
y(t)=u(t) e^{-\lambda t}=(C+o(1)) e^{-(m+\lambda) t}=(C+o(1)) e^{-t},
$$

as $t \rightarrow+\infty$, where $C>0$. Hence

$$
y(x)=(C+o(1)) \cdot x,
$$

as $x \rightarrow+0$, with some constant $C>0$.

If $a<-2$ and $2 a+\sigma+3>0$, then $B<0$ and $A<0$. From the Lemma 2.4 we have that

$$
u(t)=(C+o(1)) e^{-M t},
$$

as $t \rightarrow+\infty$, with some constant $C>0$ and $M=\sqrt{\frac{B_{1}^{2}}{4}-A}+\frac{B_{1}}{2}>0, B_{1}=-B$. In this case $M=\frac{a+\sigma+1}{\sigma-1}$. Thus, we obtain

$$
y(t)=u(t) e^{-\lambda t}=(C+o(1)) e^{-(M+\lambda) t}=(C+o(1)) e^{-t},
$$

as $t \rightarrow+\infty$, where $C>0$. Hence

$$
y(x)=(C+o(1)) \cdot x,
$$

as $x \rightarrow+0$, with some constant $C>0$. 
If $a=-2$, then $B=-1$ and $A=0$. From the Lemma 2.5 we have that

$$
u(t)=(C+o(1)) e^{-B_{1} t},
$$

as $t \rightarrow+\infty$, with some constant $C>0$ and $B_{1}=-B$. Thus, we obtain

$$
y(t)=u(t) e^{-\lambda t}=(C+o(1)) e^{-\left(B_{1}+\lambda\right) t}=(C+o(1)) e^{-t},
$$

as $t \rightarrow+\infty$, where $C>0$. Hence

$$
y(x)=(C+o(1)) \cdot x,
$$

as $x \rightarrow+0$, with some constant $C>0$;

b) If $a<-2$ and $a+\sigma+1=0$, then $A=0$ and $B=\lambda=1$. From the Lemma 2.2 we have that

$$
u(t)=((\sigma-1) t))^{-\frac{\beta}{2}}(1+o(1)),
$$

as $t \rightarrow+\infty$, where $\beta=\frac{2}{\sigma-1}>0$. Thus, we obtain

$$
y(t)=u(t) e^{-t}=(-(a+2) t)^{\frac{1}{1-\sigma}}(1+o(1)) e^{-t},
$$

as $t \rightarrow+\infty$. Hence

$$
y(x)=((a+2) \ln x)^{\frac{1}{a+2}} \cdot x \cdot(1+o(1)),
$$

as $x \rightarrow+0$.

c) If $a<-2$ and $a+\sigma+1<0$, then $A>0$ and $B>0$. Because we only look for the positive solutions of (3.1), then from Lemma 2.3 we have that

$$
u(t)=A^{\frac{\beta}{2}}+o(1),
$$

as $t \rightarrow+\infty$, where $\beta=\frac{2}{\sigma-1}>0$. Thus, we obtain

$$
y(t)=u(t) e^{-\lambda t}=\left(\left(\frac{(a+2)(a+\sigma+1)}{(1-\sigma)^{2}}\right)^{\frac{1}{\sigma-1}}+o(1)\right) e^{-\lambda t},
$$

as $t \rightarrow+\infty$. Hence

$$
y(x)=\left(\left(\frac{(a+2)(a+\sigma+1)}{(1-\sigma)^{2}}\right)^{\frac{1}{1-\sigma}}+o(1)\right) x^{\frac{a+2}{1-\sigma}},
$$

as $x \rightarrow+0$.

d) If $a>-2$, then $B<0$ and $0<A<\frac{B^{2}}{4}$. Then $m=\frac{B_{1}-1}{2}=-\lambda$ and $M=\frac{B_{1}+1}{2}=1-\lambda$. From the Lemma 2.6 follows that any non-trivial solution $u(t)$ to (2.2) tends to 0 as $t \rightarrow+\infty$ it holds

$$
u(t)=C e^{\lambda t}(1+o(1))
$$

or

$$
u(t)=C e^{(\lambda-1) t}(1+o(1)),
$$

with some non-zero constant $C$. Thus, we obtain

$$
y(t)=u(t) e^{-\lambda t}=C+o(1)
$$


or

$$
y(t)=u(t) e^{-\lambda t}=(C+o(1)) e^{-t}
$$

as $t \rightarrow+\infty$. Hence

$$
y(x)=C(1+o(1)),
$$

or

$$
y(x)=C(1+o(1)) x
$$

as $x \rightarrow+0$, with some constant $C>0$.

e) This is a solvable case and solution of differential equations (3.1) in parametric form in this case is

$$
\begin{aligned}
& x(\tau)=m C_{2}^{2} e^{2 \int\left(\frac{8}{\sigma+1} \tau^{\sigma+1}+\tau^{2}+C_{1}\right)^{-\frac{1}{2}} d \tau} \\
& y(\tau)=n C_{2} \tau e^{\int\left(\frac{8}{\sigma+1} \tau^{\sigma+1}+\tau^{2}+C_{1}\right)^{-\frac{1}{2}} d \tau},
\end{aligned}
$$

where $\left(\frac{m}{n^{2}}\right)^{\frac{\sigma-1}{2}}=1$ and $C_{1}, C_{2}$ are constants, $C_{2}>0$, and $C_{1}$ such that the expression is well-defined. This result is already known and is listed for completeness of case $\sigma>1$, and more about this case are in [6].

Corollary 3.1. If $\sigma>1$, then there exist infinitely many solutions of (3.1) defined on the interval $\left(0, x_{0}\right], x_{0}>0$, which satisfy the condition

$$
\lim _{x \rightarrow+0} y(x)=0 .
$$

Proof. Proof of corollary 3.1 follows directly from Theorem 3.1.

In the proof of Theorem 3.1 we used the relationship between the differential equation (3.1) and equations (2.1) and (2.2). The behaviour of the solutions of (2.1) and (2.2) at infinity gives us important information about the behaviour of solutions of (3.1) at zero.

Acknowledgements. This research was partly supported through Project OI174001 by the Ministry of Education, Science and Technological Development, Republic of Serbia, through Mathematical Institute SASA.

\section{REFERENCES}

[1] J. Knežević-Miljanović, Vertical asymptotes of solutions of the Emden-Fowler equation, Differ. Equ. 43 (12) (2007), 1753-1755.

[2] I. T. Kiguradze and T. A. Chanturiya, Asymptotic Properties of Solutions of Nonautonomous Ordinary Differential Equations (in Russian), Nauka, Moscow, 1990.

[3] J. Knežević-Miljanović, On the Cauchy problem for an Emden-Fowler equation, Differ. Equ. 45 (2) (2009): 267-270.

[4] I. V. Astashova, On asymptotical behavior of solutions to a quasi-linear second order differential equation, Funct. Differ. Equ. 16 (2009), 93-115.

[5] R. Bellman, Stability Theory of Differential Equations, McGraw-Hill Book Company, New York, 1953. 
[6] A. Polyanin, V. Zaitsev, Exact Solutions for Ordinary Differential Equations, Chapman \& Hall/CRC, New York, 2003.

${ }^{1}$ Department of Differential Equations,

University of Belgrade,

Studentski trg 16, 11000 Belgrade

E-mail address: marijam@matf.bg.ac.rs 